\title{
Updating a Textbook Model of Collective Behavior: Nest Wall Building in Temnothorax albipennis
}

\author{
Edith Invernizzi* and Graeme D. Ruxton \\ Centre for Biological Diversity, University of St Andrews \\ *Corresponding author (Email: ei21@st-andrews.ac.uk) \\ Citation - Invernizzi, E., \& Ruxton, G.D. (2021). Updating a textbook model of collective behavior: Nest wall \\ building in Temnothorax albipennis. Animal Behavior and Cognition, 8(2), 231-239. \\ https://doi.org/10.26451/abc.08.02.09.2021
}

\begin{abstract}
We reproduce the agent-based model of Temnothorax albipennis (formerly Leptothorax tuberointerruptus) nest building behavior described in Camazine et al. (2001) and based on the laboratory data and models found in Franks et al. (1992) and Franks and Deneubourg (1997). The model describes a set of behavioral rules that, when used by each worker independently, lead to behavioral coordination and the emergence of a coherent structure (the nest wall) without the need for advanced cognition: no direct communication between workers or explicit assessment of structural properties such as nest size is required. Some modelling details and data on the repeatability of results were missing from the original work. Here, we successfully replicate the findings and find the structure created by the model to be a good approximation of the one built under laboratory conditions. We additionally find that limited building material availability compromises the quality of the final structure under this behavioral model and that nest size might adjust to colony size over time. This latter finding appears, on the surface, to conflict with the predictions of the analytical model (Franks \& Deneubourg, 1997).
\end{abstract}

Keywords - Animal architecture, Self-organization, Ants, Collective behavior, Ant colony

In 1992, one of the first papers on Temnothorax albipennis (then Leptothorax tuberointerruptus) nest wall building also established one of the first computational models of animal collective behavior (Franks et al., 1992). The paper contained the results of a spatially-explicit agent-based model without describing its details. Later, in 1997, an analytical version of the same behavioral model was published (Franks \& Deneubourg, 1997), followed by a simpler version of the agent-based model that became, literally, a text-book example of self-organised collective behavior (Camazine et al., 2001, Chapter 17). This later version of the agent-based model is described in more detail than the one in Franks et al. (1992), but details on how to interpret the use of time in the model are missing, as well as data on the repeatability of the outcome over replicate runs of simulation. Here, we replicate this model and evaluate its ability to recreate the patterns observed in nature, also interpreting its predictions in the light of the analytical model (Franks \& Deneubourg, 1997).

The behavior described is a case of animal architecture: the wall built by workers of T. albipennis ants to protect their nest (possibly having a homeostatic function in maintaining nest temperature and humidity levels stable, as well as preventing flooding; the wall might also act as defence from other ant parasitic species in similar nests within the same genus; Camazine et al., 2001; Franks et al., 1992). This Temnothorax species nests within rock crevices or other thin openings, where it uses soil particles or other light building material to erect walls. After entering the nest, the characteristic spatial organization of this species has the cluster of queen, brood, and nurse workers in the middle of the nest and the walls approximately centred around it (Franks et al., 1992). Because of the preference of T. albipennis for 
narrow cavities, the wall is an exceptional example of nearly-2D architecture, making it a perfect case study for early computational models of collective animal building. The models (Camazine et al., 2001; Franks \& Deneubourg, 1997; Franks et al., 1992) describe individual building rules that are independently applied by each worker and lead to the construction of a coherent and functional wall structure when used by the collective, through indirect feedback mechanisms (self-organisation; Camazine et al., 2001; Invernizzi \& Ruxton, 2019; Theraulaz et al., 1998, 2003) and the use of spatial reference points (a template). Effectively, the rules explain how behavioral coordination allowing the nest wall to be built faster (through the contribution of multiple individuals) can be achieved, as well as how constant updating of nest size to respond to changes in colony size is possible. The model hypothesizes the effect of two elements on building behavior: a distance template, that affects worker pick-up and deposition probability as a function of distance from the brood cluster (centre of the nest), and a feedback effect of the number of neighboring stones on each probability function.

The agent-based model adds stochasticity and the effect of space to the behavioral rules already described by the analytical model. Therefore, the output of the agent-based model is key to evaluate how well these rules reproduce the patterns observed in nature.

\section{Method}

\section{Model Details}

We replicate the model according to the description offered in Camazine et al. (2001).

The agent-based model is time-discrete and space-discrete and contains 30 identical agents (the ant workers).

Space is designed as an $80 \times 80$ matrix - we worked bearing in mind the units used in Camazine et al. (2001), that define each node as corresponding to $1 \mathrm{~mm}$ in the physical world. Movement in space is random: from each position on the matrix, a worker can move to any other position in the next time step.

A single time step consists of one round. At each round, the following process is repeated 30 times, one for each worker. Each worker starts by moving to a new random position in the matrix and is given one chance to pick up a stone (according to probability function $P(x)$, where $x$ is the location of the worker in space; see below) from the chosen node, if there is one. If it picks up, it moves to new randomly chosen positions, one at a time, until it deposits (at each node, the probability of depositing depends on the probability function $D(x)$ ). Unless otherwise specified, we run the simulation for a total of 5,000 rounds. The use of rounds as a time unit differs from the time formulation by Camazine and co-authors, who used hours. Their time specification is unclear (and potentially misleading): if they found a means of conversion between one round of simulation and ant building time in the physical world, they do not report it; if they used computational time as a physical-world equivalent, this is not reproducible because it is dependent on machine specifications. Quite crucially, computational time in the early 90 s was several orders of magnitude slower than current standards. By specifying the time in number of rounds, we provide a comparable unit that can be used for replication and further work based on this model.

The model is initialised with stones randomly scattered throughout the matrix. Each node has a maximum carrying capacity of three stones.

\section{Behavioral Probability Functions}

We use the functions described in Camazine et al. (2001) to define the probability of pick-up, $P(x)$, and deposition, $D(x)$, where $x$ is an individual node on the matrix (i.e., a point in our simulated space). These probabilities describe a relationship between behavior and distance $r$ from the centre of the brood cluster (identified with the centre of the matrix) and the total number of stones at the chosen node and 4 neighbouring locations (i.e., the nodes North, South, East, and West of the focal node). 
The probability of pick-up is

$P(r)=P_{M}\left(1-\frac{1}{1+\tau\left(r-r_{o}\right)^{2}}\right) F(S)$

Here, $r$ is the distance of location $x$ from the centre of the brood cluster, $r_{0}$ is an optimal distance value known to workers at which the wall should be built, $S$ is the total number of stones, $P_{M}$ is the maximum possible probability of deposition if we ignore the effect of the stones and $F(S)$ is the effect of the stones:

$F(S)=F_{m}$ if $S \geq S_{c}$ and

$F(S)=F_{M}$ if $S<S_{c}$ and

$F_{m}<F_{M}$

where $S_{e}$ is a critical number of stones value after which the behavior changes.

The probability of deposition function describes the opposite relationship to distance and number of stones:

$D(x)=D_{M} \frac{1}{1+\tau\left(r-r_{o}\right)^{2}} G(S) Q$

where $Q=0$ if the maximum carrying capacity for stones at that node has already been reached and $Q=1$ otherwise. The effect of stones on deposition is:

$G(S)=G_{M}$ if $S \geq S_{c}$ and

$G(S)=G_{m}$ if $S<S_{c}$ and

$G_{m}<G_{M}$

We run all simulations with the same parameter values used in Camazine et al. (2001):

$P_{M}=0.35, G_{M}=0.5, G_{m}=F_{m}=0.01, G_{M}=F_{M}=0.55, S_{c}=6, \tau=0.025$ and $r_{o}=18$.

The distance of each matrix node to the centre was calculated by converting the matrix to cartesian coordinates (centred on the central node) and applying Pythagoras's theorem.

\section{Delayed Colony Size Increase}

The simulations testing the effect of a delayed increase in colony-size on wall location are run for 75,000 rounds. At $t=5,000, r_{0}$ is switched from 18 to 24 , to reproduce the change in template effect caused by an increase of $50 \%$ in colony size. Data are extracted at time points $t=6,000, t=10,000$ and $t$ $=75,000$. 


\section{Number of Depositions per Round}

To estimate the number of depositions in each round, we track stone positions in space round by round in a set of 20 simulations with 3,000 stones. We calculate the number of locations where the number of stones has changed, between consecutive rounds, and divide it by two to calculate the rate, because for each moved stone, both the pick-up and the deposition location change in value. Note that this value is only an estimate of the real value, because multiple stones that are moved from or to the same location are tracked only once. The average for each round is then calculated across simulations for an averaged estimate.

\section{Software}

The model was built in Python 3.7.9 (Van Rossum \& Drake, 2009) and simulations were run using Spyder 4.1.1.

\section{Statistical Measures of Stone Dispersion}

Given that the expected shape of the final wall is a circle centred around the brood cluster, we can measure the dispersion of deposition locations at the end of the simulation as dispersion over the two dimensions of a circular plane: $\theta$, the angle, and $r$, the radius (distance from the cluster). We define a deposition location as any location in the matrix that has at least one stone. We calculate dispersion along the $\theta$ axis as circular spread $\bar{R}$ (Pewsey et al., 2013):

$\bar{R}=\frac{1}{M} \sqrt[2]{\sum_{i=1}^{M} \cos \theta_{i}+\sum_{i=1}^{M} \sin \theta_{i}^{2}}$

with $M$ being all the deposition locations and $\theta_{i}$ the angle of each location $i$ measured in radians.

We measure dispersion in radius as

distance dispersion $=\frac{S D(r)}{\bar{r}}$,

the standard deviation of the distance from the centre (radius) $r$ of all $i$ divided by the average distance of these locations, $\bar{r}$.

\section{Results}

We reconstruct the agent-based model described in Camazine et al. (2001) and based on the laboratory data obtained by Franks et al. (1992) and Franks and Deneubourg (1997) as well as on the spatially explicit and on the analytical model described, respectively, in the two papers. Our results successfully replicate those of the original study, showing that the model reproduces the structural patterns made by ant behavior.

We compare the output of the model without (template only) or with (template + feedback) the effect of neighboring stones, across three different values of stone availability: 1,000, 3,000 and 5,000 stones (Figure 1A). The model including feedback best reproduces the nest wall structure built by the ants under laboratory conditions (compare with Figure 1B, Figure 1 from Franks \& Deneubourg, 1997), resulting in a more cohesive structure. We additionally observe that, when the available material is limited (1,000 stones), the feedback effect leads to the formation of local stone piles (centered at an 
optimal template-guided distance from the cluster) and prevents the formation of a complete wall, which would instead be built under a template-only mechanism. The material is not redistributed over a longer time scale (Figure 1C) and the wall remains incomplete. The wall built at low stone availability under this behavioral model is always low quality. Figure 2A, top panel, shows how dispersed deposition locations are along the distance-from-cluster axis. We use standard deviation in distance of the stones to the central cluster divided by mean distance as a measure. This definition allows the spread to be compared between behavioral models, given an equal number of stones, while simulations with higher number of stones result in higher values. We call walls with low dispersion (given equal stones) compact, meaning that they are high stone density. Figure 2A, bottom panel, shows how symmetrically distributed the angles of these locations are on a circular plane, a measure of how many gaps there are in the wall. The closer these two measures are to zero, the more symmetrical the wall and the less dispersed the stones. We can see that the more stones are present, the more variation in the distance from the cluster is visible in both models, because wall thickness increases, but (given the same building time) the template + feedback model produces a more compact wall than the template only model. However, in this model the wall formed at low stone availability is largely asymmetrical, i.e., it contains gaps.

Figure 1

Model Output Across Different Levels of Stone Availability

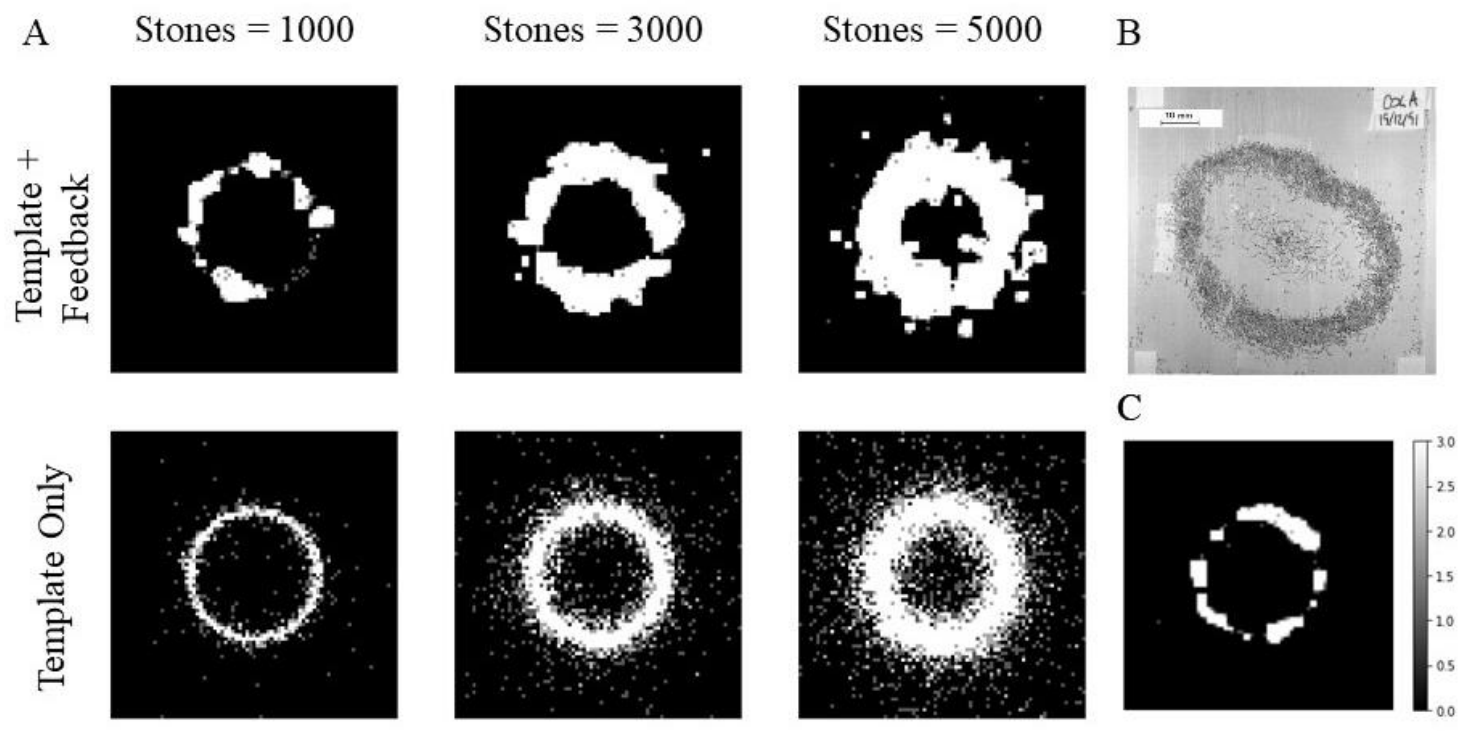

Note. Panel A shows how the output of the same simulation run changes if the model includes (top row) the feedback effect of deposited stones on future depositions and pick-ups or if it contains the template effect only (bottom row) and with different stone availabilities (this remains a constant throughout the simulation). Each simulation was run for 5,000 rounds. Panel B shows Figure 1 in Franks and Deneubourg (1997), a picture of a nest wall built by T. albipennis under laboratory conditions. Image reproduced by courtesy of N. Franks (From "Self-organising nest construction in ants: individual worker behavior and the nest's dynamics," by N. R. Franks and J-L. Deneubourg, 1997, Animal Behavior, 54(4), 779-796. Elsevier. Reprinted with permission. Panel C shows the output of the same run as the top left subplot of panel A, after 20,000 rounds. The grayscale reflects the number of stones at each location (corresponding numerical scale to the right of the figure).

T. albipennis colonies also fail to adjust nest size when colony size is suddenly doubled (by reintroducing the previously removed half of the colony) after a complete wall has already been built (Franks \& Deneubourg, 1997). The template + feedback model is shown, in the original work, to successfully reproduce this effect (Camazine et al., 2001). When replicating these simulations, we find that this is true, but only over short-term time scales (Figures $3 \mathrm{~A}$ and $3 \mathrm{~B}$, first panel). If we observe the wall for a longer time after reintroduction of the missing colony half, we see it progressively move outwards, approaching the new expected nest size (Figures $3 \mathrm{~A}$ and 3B, second and third panels). 
The lack of adjustment of nest area to population size observed in real colonies is the effect of hysteresis, the existence of multiple stationary states given the same parameters, where the final state reached is due to the history of the system (this is better explained in the analytical model presented in Franks \& Deneubourg, 1997). Why, then, do our simulations show progressive expansion? The results from this model do not contradict real data, nor the Franks and Deneubourg (1997) model. While, on average, the wall is stable (that is, deposition and pick-up activity are balanced), complex systems are noisy, resulting in local imbalanced activity. Because the amount of building material is finite, over time, this noise causes continuous relocation of material towards the location in the nest that is now favored by the template. The wall slowly moves towards the new optimal value. This means that, in real systems and given enough time (and the same template effect as used in the model), we expect the wall to slowly expand to accommodate the larger colony.

\section{Figure 2}

Final Stone Distribution

A

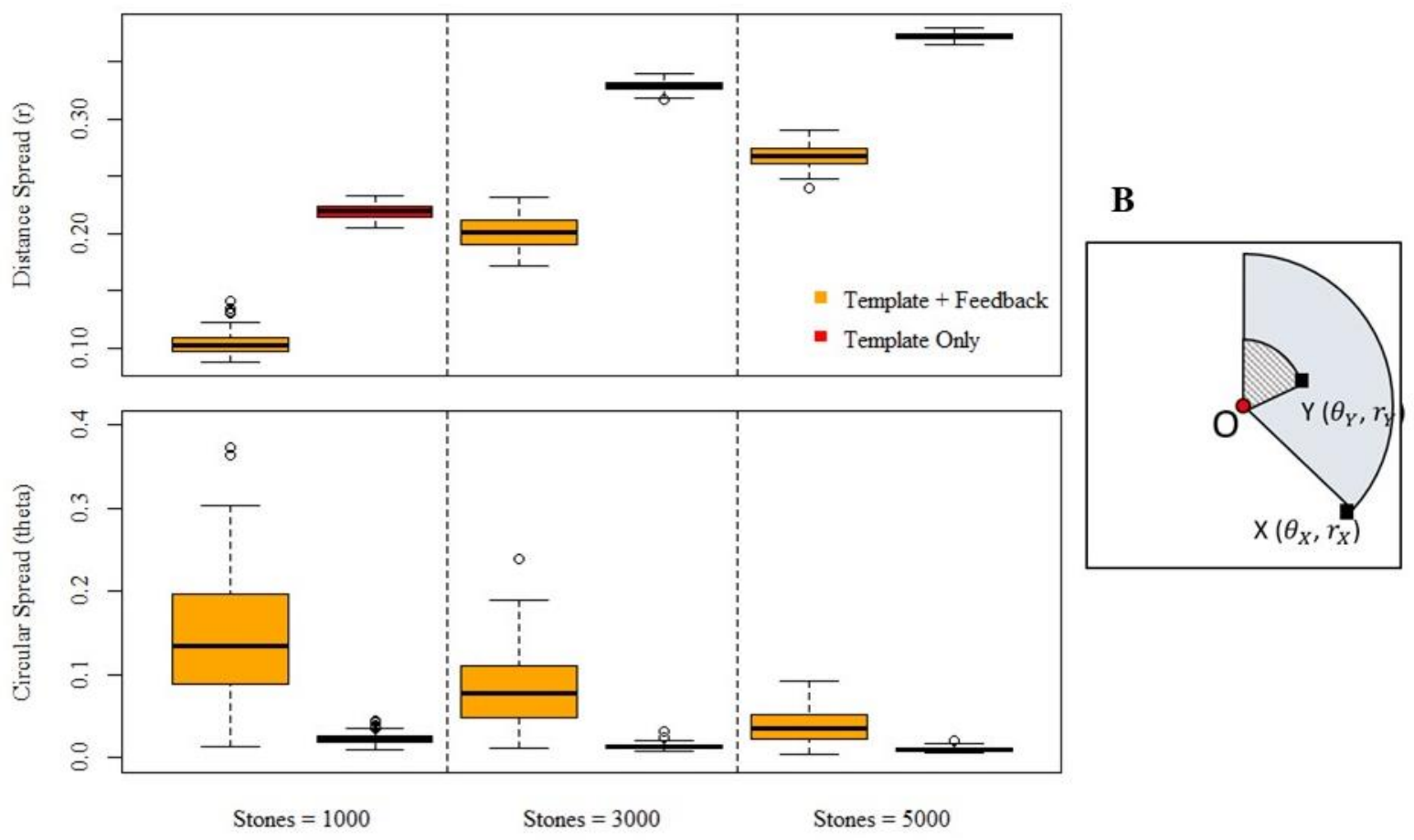

Note. Panel A shows how the spread of building locations on the two axes of a circular plane varies over 100 simulations. The two axes are: distance from the cluster (distance spread or spread in $r$; top panel) and angular axis (circular spread or spread in $\boldsymbol{\theta}$; bottom panel). Details of the statistical measures used are found in Methods. Panel B shows what $r$ and $\boldsymbol{\theta}$ represent. Two deposition locations, $\mathrm{X}$ and $\mathrm{Y}$, can be represented on a circular plane (with origin in the brood cluster $\mathrm{O}$ ) using two coordinates: the distance from the cluster $r$ and the angle $\boldsymbol{\theta}$, measured from 12 o'clock.

We can attempt to convert computational time into estimated real time. Time conversion of this type of simulation should be treated with extreme caution: the goal of the simulation is to assess, qualitatively, whether the behavioral paradigm reproduced can generate walls similar to the one observed in nature, and not to accurately reproduce the system. This means that some simulation details are unrealistic when looked at from a time perspective. In addition, the model does not incorporate details of the system that do not modify the qualitative outcome but may alter its time scale. Activity cycles in ant colonies (Cole, 1991), for example, have a key influence on the overall activity rate, including building rate, so that rates calculated over short term periods differ from those calculated over the full length of the 
building activity. Franks and Deneubourg (1997) provide a "“global' rate of stone deposition" (p. 784), but do not offer details on how this is calculated, or on the number of building workers in the colonies, so that we do not know how this may compare to our simulation. While attempting a conversion here, this should only be considered a rough estimate with no pretension of accuracy. Franks and Deneubourg (1997) state that a wall is approximately completed in $24 \mathrm{hrs}$. In our simulations, the estimated number of depositions per round levels to almost zero around the 2,000th round (Figure 4). We can consider this point as the time point in the model at which the wall is completed (i.e., the system reaches a point of equilibrium after which pick-ups and deposition are balanced in both number and location and the wall is not substantially altered), meaning that the correspondence between one round and real time is approx. 43 s/round. Therefore, in Figure 3, the first time point (6,000 rounds) corresponds to $12 \mathrm{hrs}$ after population increase. At this stage, we see little change in next size. The second and third time points $(10,000$ and 75,000 rounds) correspond to 4.5 and 37 days after population increase, respectively. This indicates that accommodation of the wall to the new equilibrium point is a slow process.

\section{Figure 3}

Shift to New Nest Size Over Time

A

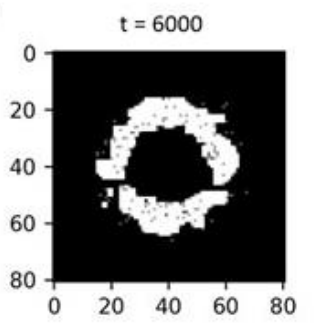

$t=10000$

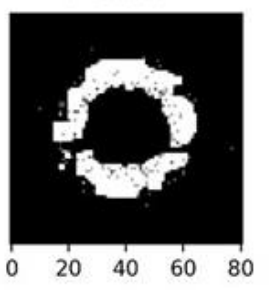

$t=75000$

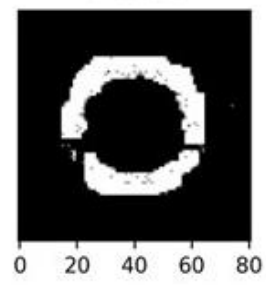

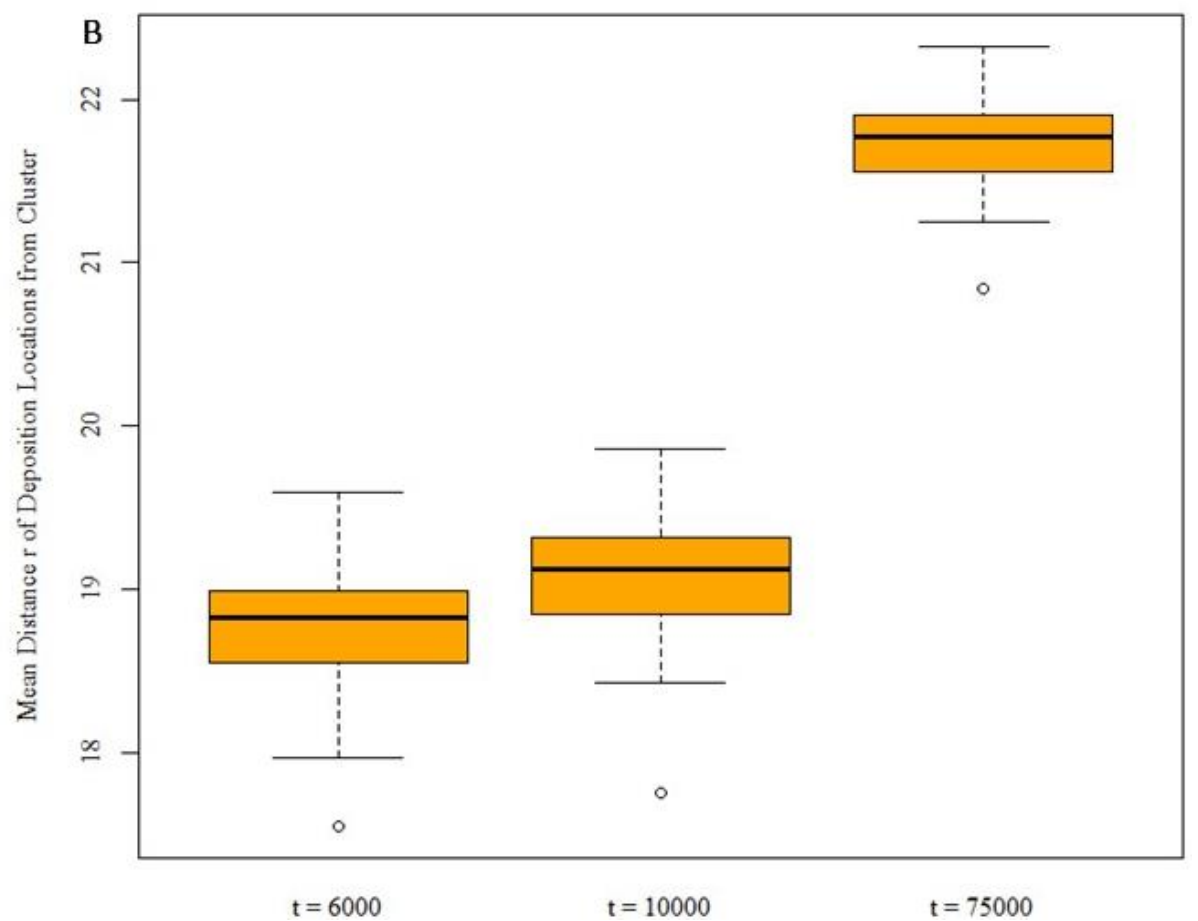

Note. The figure shows what happens to the wall given enough time after colony size increase. Simulations are run for 75,000 time steps, starting with $\mathrm{r}_{0}=18$ and switching to $\mathrm{r}_{0}=24$ at time step 5,000. In panel A, three sample outputs from three time points in the simulation, $t=6,000$ (corresponding to short-term change after a population increase - see main text), $t=10,000$ and $t=75,000$, are shown. On the axes, each unit corresponds to one node in simulated space, $1 \mathrm{~mm}$ in real space. The centre of the cluster is modelled to be at coordinates $(41,41)$. Panel B shows the distribution of the mean distance $r$ of deposition locations from the centre over 100 simulation runs, at the same time points. 
Figure 4

Average Colony Deposition Rate Across Simulation Time

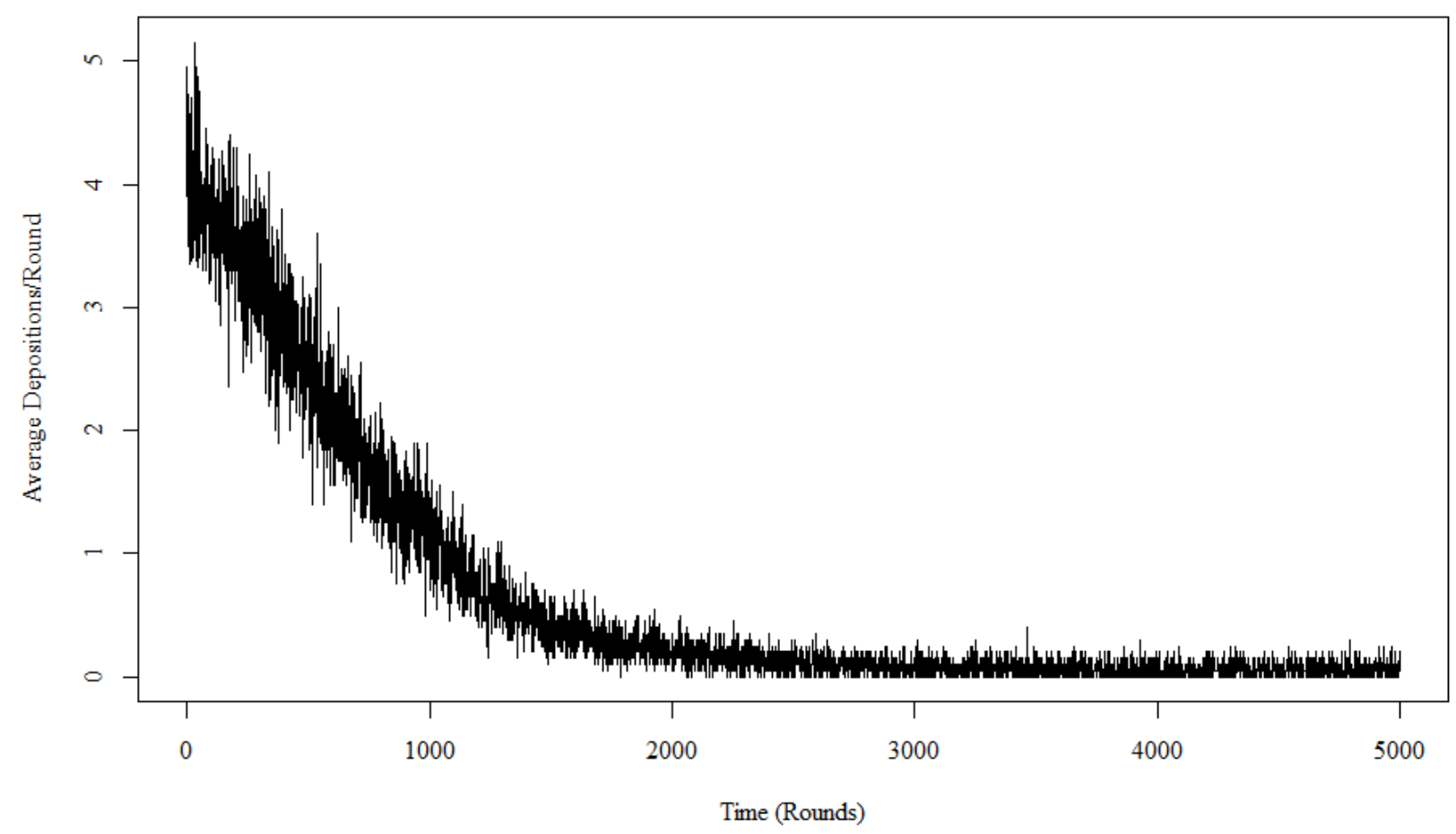

Note. The figure shows the estimated number of depositions per round occurring in the simulations, averaged across 20 runs. Simulations are run with 3,000 stones, for 5,000 rounds.

\section{Discussion}

We show here that the model presented in Camazine et al. (2001) and based on Franks et al. (1992) and Franks and Deneubourg (1997) successfully reproduces two key characteristics of $T$. albipennis nest wall building: shape of the wall and hysteresis. We find the results of this agent-based model representation of the system to be in agreement with the analytical description contained in Franks and Deneubourg (1997). Additionally, we highlight two model predictions missing from the original work: 1 . when building material is scarce, the wall will remain incomplete; and 2. over time, nest size will always adjust to current colony size, even under hysteresis conditions.

The failure of the behavioral model to create a functional wall at low stone availability does not necessarily conflict with observation. Crevice nesting Temnothorax species do not build walls if preexisting barriers are available, but rather use wall building to fill gaps (T. albipennis, Franks et al., 1992; Temnothorax rugatulus, personal observation). This behavior fits the hypothesis of an adaptive solution to avoid wasting building material. Alternatively, the deleterious consequences of a strong positive feedback can be avoided if the direction of stone deposition is guided (in combination with or instead of the use of the brood cluster as a template) by an environmental template such as the presence of air drafts. Such a template should decrease deposition in areas where a partial wall is already present and encourage stone redistribution to form a complete circular wall. Finally, a third possibility is that this in an incomplete behavioral model: the authors of the original work highlight that the model is based on worker behavior observed at the beginning of the activity (Camazine et al., 2001; Franks et al., 1992). It is possible that additional behavioral rules are used later in the process and lead to wall gaps being filled. Experimental investigations are necessary to resolve this point.

The hysteresis effect seen in experiments (Franks \& Deneubourg, 1997) is observed over a shortterm period and there are no data on change in colony size in the long term. Repeating the experiment and 
maintaining colony size stable for several weeks after the sudden increase (by removing newly developed ants unless they replace dead workers, for example) would enable testing the predictions of the model. In natural settings (in the absence of colony-size control), the tendency of the wall to expand over time only becomes relevant if colony growth rate is slow compared to building rate at equilibrium. If the colony grows (or shrinks) quickly, then a bout of intense reconstruction is initiated to accommodate nest size.

Author contributions: Concept: EI and GDR; modelling: EI; statistics: EI and GDR; manuscript and figures: EI.

Conflict of interest: The authors declare no conflict of interest.

\section{Acknowledgements}

Thanks to Tucker R. Gilman for a useful discussion of the new findings.

\section{References}

Cole, B. J. (1991). Is animal behavior chaotic? Evidence from the activity of ants. Proceedings of the Royal Society B: Biological Sciences, 244(1311), 253-259. https://www.jstor.org/stable/76608

Camazine, S., Deneubourg, J.-L., Franks, N. R., Sneyd, J., Theraulaz, G., \& Bonabeau, E. (2001). Self-organization in biological systems. Princeton Studies in Complexity.

Franks, N. R., \& Deneubourg, J. L. (1997). Self-organizing nest construction in ants: Individual worker behavior and the nest's dynamics. Animal Behavior, 54(4), 779-796. https://doi.org/10.1006/anbe.1996.0496

Franks, N. R., Wilby, A., Silverman, B. W., \& Tofts, C. (1992). Self-organizing nest construction in ants: Sophisticated building by blind bulldozing. Animal Behavior, 44(Part 2), 357-375. https://doi.org/10.1016/0003-3472(92)90041-7

Invernizzi, E., \& Ruxton, G. D. (2019). Deconstructing collective building in social insects: Implications for ecological adaptation and evolution. Insectes Sociaux, 66(4), 507-518. https://doi.org/10.1007/s00040-01900719-7

Pewsey, A., Neuhäuser, M., \& Ruxton, G. D. (2013). Circular statistics in R (First). Oxford University Press.

Theraulaz, G., Bonabeau, E., \& Deneubourg, J.-L. (1998). The origin of nest complexity in social insects learning from the models of nest construction. Complexity, 3(6). https://doi.org/10.1002/(SICI)10990526(199807/08)3:6<15::AID-CPLX3>3.0.CO;2-V

Theraulaz, G., Gautrais, J., Camazine, S., \& Deneubourg, J.-L. (2003). The formation of spatial patterns in social insects: From simple behaviors to complex structures. Philosophical Transactions of the Royal Society A: Mathematical, Physical and Engineering Sciences, 361(1807), 1263-1282. https://doi.org/10.1098/rsta.2003.1198

Van Rossum, G., \& Drake, F. L. (2009). Python 3 reference manual. CreateSpace. 